\title{
INVERSE PROBLEMS FOR DIFFERENTIAL OPERATORS WITH NONSEPARATED BOUNDARY CONDITIONS IN THE CENTRAL SYMMETRIC CASE
}

\author{
VJACHESLAV YURKO
}

\begin{abstract}
Inverse spectral problems for Sturm-Liouville operators on a finite interval with non-separated boundary conditions are studied in the central symmetric case, when the potential is symmetric with respect to the middle of the interval. We discuss statements of the problems, provide algorithms for their solutions along with necessary and sufficient conditions for the solvability of the inverse problems considered.
\end{abstract}

\section{Introduction}

We study inverse spectral problems for the Sturm-Liouville operator

$$
\ell y:=y^{\prime \prime}+q(x) y, x \in(0, \pi)
$$

on the finite interval $(0, \pi)$ with non-separated boundary conditions. Inverse problems consist in recovering coefficients of differential operators from their spectral characteristics. Such problems often appear in mathematics, mechanics, physics, geophysics, electronics and other branches of natural sciences and engineering. Inverse problems also play an important role in solving nonlinear evolution equations in mathematical physics. Inverse problems for differential operators with separated boundary conditions have been studied fairly completely by many authors (see the monographs [1]-[5] and the references therein). Inverse problems for Sturm-Liouville operators with non-separated boundary conditions, which are more difficult for the investigation, were treated in [6]-[17] and other works. In particular, the periodic boundary value problem was considered in [6, 7, 9, 14]. Stankevich [6] suggested a statement of the inverse problem and proved the corresponding uniqueness theorem. Marchenko and Ostrovskii [7] gave the characterization of the spectrum for the periodic boundary value problem in terms of a special conformal mapping. Conditions considered in [7] are difficult

Received May 31, 2017, accepted June 28, 2017.

2010 Mathematics Subject Classification. Primary 34A55; Secondary 34L05, 47E05.

Key words and phrases. Differential operators, non-separated boundary conditions, inverse spectral problems. 
to verify. Another method, used in [9], allowed to obtain necessary and sufficient conditions for the solvability of the inverse problem for the periodic case that are easier to verify. Similar results were obtained in [9] for another type of boundary conditions, namely

$$
y^{\prime}(0)-a y(0)+b y(\pi)=y^{\prime}(\pi)+d y(\pi)-b y(0)=0 .
$$

Later analogous results were established in [12, 13].

In this paper we study the case when the potential $q$ is symmetric with respect to the middle of the interval, i.e. $q(x)=q(\pi-x)$ a.e. on $(0, \pi)$. The symmetric case requires nontrivial modifications in the method and allows us to specify less spectral information than in the general case. Some results for the symmetric case were obtained in [10] and [17]. In the present paper for the symmetric case we construct the solution of the inverse spectral problem and give the characterization of the spectrum for various non-separated boundary conditions. For convenience of readers in Section 2 we describe briefly the known results for the general (non-symmetric) case.

\section{Periodic boundary value problem}

Consider the differential equation

$$
-y^{\prime \prime}+q(x) y=\lambda y, \quad x \in(0, \pi)
$$

where $\lambda$ is the spectral parameter, and $q(x) \in L_{2}(0, T)$ is a real-valued function. The function $q(x)$ is called the potential. Let $C(x, \lambda), S(x, \lambda)$ and $\psi(x, \lambda)$ be solutions of Eq. (1) with the initial conditions $C(0, \lambda)=S^{\prime}(0, \lambda)=-\psi^{\prime}(\pi, \lambda)=1, C^{\prime}(0, \lambda)=S(0, \lambda)=\psi(\pi, \lambda)=0$. For each fixed $x$, the functions $C^{(v)}(x, \lambda), S^{(v)}(x, \lambda)$ and $\psi^{(v)}(x, \lambda), v=0,1$, are entire in $\lambda$ of order $1 / 2$. Moreover,

$$
\langle C(x, \lambda), S(x, \lambda)\rangle \equiv 1,
$$

where $\langle y, z\rangle:=y z^{\prime}-y^{\prime} z$ is the Wronskian of $y$ and $z$. Denote

$$
\Delta(\lambda)=\left(C(\pi, \lambda)+S^{\prime}(\pi, \lambda)\right) / 2, \delta(\lambda)=\left(C(\pi, \lambda)-S^{\prime}(\pi, \lambda)\right) / 2, p(\lambda)=1-\Delta(\lambda) .
$$

Zeros $\Lambda=\left\{\lambda_{n}\right\}_{n \geq 0}$ of the entire function $p(\lambda)$ coincide with the eigenvalues of the boundary value problem (BVP) $L=L(q)$ for Eq. (1) with periodic boundary conditions

$$
y(0)-y(\pi)=y^{\prime}(0)-y^{\prime}(\pi)=0 .
$$

The function $p(\lambda)$ is called the characteristic function for $L$. For convenience of readers we describe briefly the well-known results related to the BVP $L$ (see $[6,7,9]$ for details). 
(1) All eigenvalues $\lambda_{n}$ are real, and

$$
\begin{aligned}
& \lambda_{0}<\lambda_{1} \leq \lambda_{2}<\lambda_{3} \leq \lambda_{4}<\ldots, \\
\lambda_{2 n}= & (2 n)^{2}+\alpha+\kappa_{2 n}, \lambda_{2 n-1}=(2 n)^{2}+\alpha+\kappa_{2 n-1}, \quad\left\{\kappa_{n}\right\} \in l_{2},
\end{aligned}
$$

where $\alpha=\frac{1}{\pi} \int_{0}^{\pi} q(t) d t$. Here and everywhere below one and the same symbol $\left\{\kappa_{n}\right\}$ denotes various sequences from $l_{2}$. The specification of $\Lambda$ uniquely determines the characteristic function $p(\lambda)$ by the formula

$$
p(\lambda)=\frac{\pi^{2}}{2}\left(\lambda-\lambda_{0}\right) \prod_{n=1}^{\infty} \frac{\lambda_{2 n}-\lambda}{(2 n)^{2}} \prod_{n=1}^{\infty} \frac{\lambda_{2 n-1}-\lambda}{(2 n)^{2}} .
$$

Moreover,

$$
\max _{\lambda \in\left[\lambda_{2 n}, \lambda_{2 n+1}\right]} p(\lambda) \geq 2, \quad n \geq 0 .
$$

(2) Let $\Lambda^{+}=\left\{\lambda_{n}^{+}\right\}_{n \geq 1}$ be zeros of the entire function $p^{+}(\lambda):=p(\lambda)-2$. Then $\left\{\lambda_{n}^{+}\right\}_{n \geq 0}$ are real and

$$
\begin{gathered}
\lambda_{0}<\lambda_{1}^{+} \leq \lambda_{2}^{+}<\lambda_{1} \leq \lambda_{2}<\lambda_{3}^{+} \leq \lambda_{4}^{+}<\lambda_{3} \leq \lambda_{4} \ldots, \\
\lambda_{2 n}^{+}=(2 n-1)^{2}+\alpha+\kappa_{2 n}, \lambda_{2 n-1}^{+}=(2 n-1)^{2}+\alpha+\kappa_{2 n-1}, \quad\left\{\kappa_{n}\right\} \in l_{2} .
\end{gathered}
$$

Denote $a_{2 n}=\left[\lambda_{2 n-1}, \lambda_{2 n}\right], a_{2 n-1}=\left[\lambda_{2 n-1}^{+}, \lambda_{2 n}^{+}\right], n \geq 1$. Segments $a_{n}$ are called the gaps.

(3) Denote $d(\lambda):=\langle\psi(x, \lambda), S(x, \lambda)\rangle=S(\pi, \lambda)=\psi(0, \lambda)$. Then zeros $\gamma=\left\{\gamma_{n}\right\}_{n \geq 1}$ of the entire function $d(\lambda)$ coincide with the eigenvalues of the BVP $L_{0}=L_{0}(q)$ for Eq. (1) with Dirichlet boundary conditions $y(0)=y(\pi)=0$. The numbers $\gamma_{n}$ are real, $\gamma_{n} \in a_{n}$, and

$$
\gamma_{1}<\gamma_{2}<\gamma_{3}<\cdots ; \quad \gamma_{n}=n^{2}+\alpha+\kappa_{n},\left\{\kappa_{n}\right\} \in l_{2} .
$$

The specification of $\gamma$ uniquely determines the characteristic function $d(\lambda)$ of $L_{0}$ by the formula

$$
d(\lambda)=\pi \prod_{n=1}^{\infty} \frac{\gamma_{n}-\lambda}{n^{2}} .
$$

The numbers $\alpha_{n}:=\int_{0}^{\pi} S^{2}\left(x, \gamma_{n}\right) d x$ are called the weight numbers, and numbers $\left\{\gamma_{n}, \alpha_{n}\right\}_{n \geq 1}$ are called the spectral data for the BVP $L_{0}$. One has

$$
\begin{aligned}
\alpha_{n} & =\dot{d}\left(\gamma_{n}\right) S^{\prime}\left(\pi, \gamma_{n}\right), \quad \dot{d}(\lambda):=\frac{d}{d \lambda} d(\lambda), \\
\alpha_{n}>0 ; \quad \alpha_{n} & =\frac{\pi}{2 n^{2}}\left(1+\frac{\kappa_{n}}{n}\right), \quad\left\{\kappa_{n}\right\} \in l_{2}, \\
\dot{d}\left(\gamma_{n}\right) & =\frac{(-1)^{n} \pi}{2 n^{2}}\left(1+\frac{\kappa_{n}}{n}\right), \quad\left\{\kappa_{n}\right\} \in l_{2}, \quad \operatorname{sign} \dot{d}\left(\gamma_{n}\right)=(-1)^{n} .
\end{aligned}
$$

The functions $S\left(x, \gamma_{n}\right)$ and $\psi\left(x, \gamma_{n}\right)$ are eigenfunctions for $L_{0}$, and

$$
\psi\left(x, \gamma_{n}\right)=\beta_{n} S\left(x, \gamma_{n}\right), \quad \beta_{n} \neq 0 .
$$


Lemma 1. The following relation holds

$$
\alpha_{n} \beta_{n}=-\dot{d}\left(\gamma_{n}\right)
$$

Proof. Since

$$
-\psi^{\prime \prime}(x, \lambda)+q(x) \psi(x, \lambda)=\lambda \psi(x, \lambda),-S^{\prime \prime}\left(x, \gamma_{n}\right)+q(x) S\left(x, \gamma_{n}\right)=\gamma_{n} S\left(x, \gamma_{n}\right) \text {, }
$$

we get

$$
\frac{d}{d x}\left\langle\psi(x, \lambda), S\left(x, \gamma_{n}\right)\right\rangle=\left(\lambda-\gamma_{n}\right) \psi(x, \lambda) S\left(x, \gamma_{n}\right)
$$

and hence,

$$
\left(\lambda-\gamma_{n}\right) \int_{0}^{\pi} \psi(x, \lambda) S\left(x, \gamma_{n}\right) d x=\left.\left\langle\psi(x, \lambda), S\left(x, \gamma_{n}\right)\right\rangle\right|_{0} ^{\pi}=-d(\lambda) .
$$

For $\lambda \rightarrow \lambda_{n}$, this yields

$$
\int_{0}^{\pi} \psi\left(x, \gamma_{n}\right) S\left(x, \gamma_{n}\right) d x=-\dot{d}\left(\gamma_{n}\right)
$$

Using (14) we arrive at (15).

The inverse problem for the BVP $L_{0}$ is formulated as follows.

Inverse problem 1. Given the spectral data $\left\{\gamma_{n}, \alpha_{n}\right\}_{n \geq 1}$, construct the potential $q$.

This inverse problem is related to the case of separated boundary conditions. It is known that the specification of the spectral data $\left\{\gamma_{n}, \alpha_{n}\right\}_{n \geq 1}$ uniquely determines the potential $q$. The global solution of Inverse problem 1 can be constructed by the transformation operator method or by the method of spectral mappings (see [1]-[5] for details). In particular, these methods allow one to describe necessary and sufficient conditions for the solvability of Inverse problem 1 which are presented in the next theorem.

Theorem 1. For real numbers $\left\{\gamma_{n}, \alpha_{n}\right\}_{n \geq 1}$ to be the spectral data for a certain BVP $L_{0}$ with a real potential $q(x) \in L_{2}(0, \pi)$, it is necessary and sufficient that (9) and (12) hold.

Let us now return to the periodic BVP $L$. It follows from (2) that

$$
\Delta^{2}(\lambda)-\delta^{2}(\lambda)-d(\lambda) d_{1}(\lambda) \equiv 1
$$

where $d_{1}(\lambda):=C^{\prime}(\pi, \lambda)$. In particular, (16) yields

$$
\delta^{2}\left(\gamma_{n}\right)=\Delta^{2}\left(\gamma_{n}\right)-1
$$

Denote $\Omega=\left\{\omega_{n}\right\}_{n \geq 1}, \omega_{n}=\operatorname{sign} \delta\left(\gamma_{n}\right)$. The sequence $\Omega$ is called the $\Omega$ - sequence for $q$. In view of (17) one has

$$
\delta\left(\gamma_{n}\right)=\omega_{n}\left(\Delta^{2}\left(\gamma_{n}\right)-1\right)^{1 / 2}
$$


Since $S^{\prime}\left(\pi, \gamma_{n}\right)=\Delta\left(\gamma_{n}\right)-\delta\left(\gamma_{n}\right)$, it follows from (11) and (18) that

$$
\alpha_{n}=\dot{d}\left(\gamma_{n}\right)\left(\Delta\left(\gamma_{n}\right)-\omega_{n}\left(\Delta^{2}\left(\gamma_{n}\right)-1\right)^{1 / 2}\right) .
$$

The inverse problem for the periodic case is formulated as follows [6].

Inverse problem 2. Given $\Lambda, \gamma$ and $\Omega$, construct the potential $q$.

This inverse problem was studied in $[6,7,9,14]$ and other works. It was proved in [6] that the specification of $\Lambda, \gamma$ and $\Omega$ uniquely determines the potential $q$. In order to construct $q$ one can calculate the functions $p(\lambda)$ and $d(\lambda)$ according to (5) and (10), and construct $\left\{\alpha_{n}\right\}_{n \geq 1}$ via (19), where $\Delta(\lambda)=1-p(\lambda)$. Then using data $\left\{\gamma_{n}, \alpha_{n}\right\}_{n \geq 1}$, we can construct the potential $q$ by solving Inverse problem 1.

Lemma 2. Fix $n \geq 1$. Relation $\delta\left(\gamma_{n}\right)=0$ holds iff $\gamma_{n}$ lies at one of the endpoints of the gap $a_{n}$.

Indeed, in view of (17), $\delta\left(\gamma_{n}\right)=0$, iff $\Delta\left(\gamma_{n}\right)= \pm 1$, i.e. $\gamma_{n}$ lies at one of the endpoints of the gap $a_{n}$.

Denote by $J$ the set of sequences $\Omega=\left\{\omega_{n}\right\}_{n \geq 1}$ such that $\omega_{n}=0$ if $\gamma_{n}$ lies at one of the endpoints of the gap $a_{n}$, and $\omega_{n}= \pm 1$, otherwise. Clearly, if $\Omega$ is the $\Omega$ - sequence for $L$, then $\Omega \in J$. The next theorem [9] establishes necessary and sufficient conditions for the solvability of Inverse problem 2.

Theorem 2 ([9]). Let real numbers $\Lambda=\left\{\lambda_{n}\right\}_{n \geq 0}$ satisfying (3)-(4) be given. The sequence $\Lambda$ is the spectrum for a certain BVP L with a real potential $q(x) \in L_{2}(0, \pi)$, iff relation (6) holds, where $p(\lambda)$ is constructed via (5). Moreover, if additionally we have a sequence $\gamma=\left\{\gamma_{n}\right\}_{n \geq 1}, \gamma_{n} \in a_{n}$, satisfying (9), where $\Lambda^{+}=\left\{\lambda_{n}^{+}\right\}_{n \geq 1}$ are zeros of $p^{+}(\lambda)=p(\lambda)-2$, and a sequence $\Omega=\left\{\omega_{n}\right\}_{n \geq 1} \in J$, then there exists a unique real function $q(x) \in L_{2}(0, \pi)$ such that $\Lambda$ and $\gamma$ are the spectra of $L$ and $L_{0}$, respectively, and $\Omega$ is the $\Omega$ - sequence for $L$.

The next theorem [9] shows that one of the endpoints of each gap can be chosen arbitrary taking only asymptotics into account.

Theorem 3 ([9]). Let real numbers $\theta_{n}$ of the form $\theta_{n}=n^{2}+\alpha+\kappa_{n},\left\{\kappa_{n}\right\} \in l_{2}, \theta_{n}<\theta_{n+1}$, be given. Then there exists a real function $q(x) \in L_{2}(0, \pi)$ (not unique!) such that for this potential the number $\theta_{n}$ lies at one of the endpoints of the gap $a_{n}$ for all $n \geq 1$.

\section{Central symmetric case.}

In this section we consider the case when the potential $q$ is symmetric with respect to the middle of the interval, i.e. with respect to the replacement $x \rightarrow \pi-x$. We will say that $q(x) \in L_{2}^{\prime}(0, \pi)$ if $q(x) \in L_{2}(0, \pi)$ and $q(x)=q(\pi-x)$ a.e. on $(0, \pi)$. 
Theorem 4. $q(x) \in L_{2}^{\prime}(0, \pi)$ iff $\beta_{n}=(-1)^{n-1}, n \geq 1$.

\section{Proof.}

(1) Let $q(x) \in L_{2}^{\prime}(0, \pi)$. Then $\psi(x, \lambda) \equiv S(\pi-x, \lambda)$. Using (14) we calculate

$$
\psi\left(x, \gamma_{n}\right)=\beta_{n} S\left(x, \gamma_{n}\right)=\beta_{n} \psi\left(\pi-x, \gamma_{n}\right)=\beta_{n}^{2} S\left(\pi-x, \gamma_{n}\right)=\beta_{n}^{2} \psi\left(x, \gamma_{n}\right) .
$$

Hence, $\beta_{n}^{2}=1$. On the other hand, it follows from (14) that $\beta_{n} S^{\prime}\left(\pi, \gamma_{n}\right)=-1$. Using Sturm's oscillation theorem we conclude that $\beta_{n}=(-1)^{n-1}, n \geq 1$.

(2) Let $\beta_{n}=(-1)^{n-1}, n \geq 1$. Denote $\tilde{q}(x):=q(\pi-x)$. We agree that here and below, if a certain symbol $\theta$ denotes an object related to $q$, then $\tilde{\theta}$ will denote the analogous object related to $\tilde{q}$.

Obviously, $\tilde{\psi}(x, \lambda) \equiv S(\pi-x, \lambda), \tilde{S}(x, \lambda) \equiv \psi(\pi-x, \lambda)$, and consequently, $d(\lambda) \equiv \tilde{d}(\lambda)$ and $\gamma_{n}=\tilde{\gamma}_{n}, n \geq 1$. Since $\beta_{n}=(-1)^{n-1}$, it follows from (14) that $\psi\left(x, \gamma_{n}\right)=(-1)^{n-1} S\left(x, \gamma_{n}\right)$. Moreover, according to (14), $\tilde{\psi}\left(x, \gamma_{n}\right)=\tilde{\beta}_{n} \tilde{S}\left(x, \gamma_{n}\right)$, hence $S\left(\pi-x, \gamma_{n}\right)=\tilde{\beta}_{n} \psi\left(\pi-x, \gamma_{n}\right)$, i.e. $\tilde{\beta}_{n}=$ $\left(\beta_{n}\right)^{-1}=(-1)^{n-1}$. Thus, $\beta_{n}=\tilde{\beta}_{n}$ for all $n \geq 1$. Taking (15) into account we conclude that $\alpha_{n}=\tilde{\alpha}_{n}$ for all $n \geq 1$. Since the specification of the spectral data $\left\{\gamma_{n}, \alpha_{n}\right\}_{n \geq 1}$ uniquely determines the potential, we obtain that $q(x)=\tilde{q}(x)$ a.e. on $(0, \pi)$, i.e. $q(x) \in L_{2}^{\prime}(0, \pi)$.

Let us consider the inverse problem for the BVP $L_{0}$. In the central symmetric case $q(x) \in$ $L_{2}^{\prime}(0, \pi)$ we do not need to specify the weight numbers $\left\{\alpha_{n}\right\}_{n \geq 1}$; it is sufficient to specify only the spectrum $\gamma$.

Inverse problem 3. Given the spectrum $\gamma=\left\{\gamma_{n}\right\}_{n \geq 1}$, construct the potential $q$.

It is known [1]-[5] that for the central symmetric case the specification of the spectrum $\gamma=\left\{\gamma_{n}\right\}_{n \geq 1}$ of the BVP $L_{0}$ uniquely determines the potential $q$. In order to construct $q$, one can calculate $d(\lambda)$ via (10) and the weight numbers $\alpha_{n}=(-1)^{n} \dot{d}\left(\gamma_{n}\right)$, and then find $q$ by solving Inverse problem 1. Moreover, the characterization of the spectrum of $L_{0}$ is given by the following assertion.

Theorem 5. For real numbers $\left\{\gamma_{n}\right\}_{n \geq 1}$ to be the spectrum of a BVP $L_{0}$ with a real potential $q(x) \in L_{2}^{\prime}(0, \pi)$, it is necessary and sufficient that (9) holds.

Proof. The necessity is obvious. We will prove the sufficiency. Let real numbers $\left\{\gamma_{n}\right\}_{n \geq 1}$ satisfying (9) be given. We construct $d(\lambda)$ via (10) and the numbers $\left\{\alpha_{n}\right\}_{n \geq 1}$ by $\alpha_{n}=(-1)^{n} \dot{d}\left(\gamma_{n}\right)$. Our plan is to use Theorem 1. For this purpose we should obtain the asymptotics for the numbers $\alpha_{n}$. This seems to be difficult because the function $d(\lambda)$ is by construction the infinite product. But fortunately, for calculating the asymptotics of $\alpha_{n}$ one can also use 
Theorem 1, as an auxiliary assertion. Indeed, by virtue of Theorem 1 there exists a potential $\tilde{q}(x) \in L_{2}(0, \pi)$ (not unique) such that $\gamma=\left\{\gamma_{n}\right\}_{n \geq 1}$ is the spectrum of $\tilde{L}_{0}:=L_{0}(\tilde{q})$ with this potential. Then $d(\lambda)$ is the characteristic function of $\tilde{L}_{0}$, and consequently, (13) holds. Therefore, (12) is valid. Then, by Theorem 1 there exists a unique potential $q(x) \in L_{2}(0, \pi)$ such that $\left\{\gamma_{n}, \alpha_{n}\right\}_{n \geq 1}$ are the spectral data of $L_{0}(q)$. Since $\beta_{n}=(-1)^{n-1}, n \geq 1$, it follows from Theorem 4 that $q(x) \in L_{2}^{\prime}(0, \pi)$.

Theorem 6 ([9]). $q(x) \in L_{2}^{\prime}(0, \pi)$ iff $\gamma_{n}$ lies at one of the endpoints of the gap $a_{n}$ for all $n \geq 1$.

\section{Proof.}

(1) Let $q(x)=q(\pi-x)$ a.e. on $(0, \pi)$. Using Lemma 4 from [8] we get $C(\pi, \lambda) \equiv S^{\prime}(\pi, \lambda)$, i.e. $\delta(\lambda) \equiv 0$. By Lemma 2 we conclude that $\gamma_{n}$ lies at one of the endpoints of the gap $a_{n}$ for all $n \geq 1$.

(2) Let $\gamma_{n}$ lie at one of the endpoints of the gap $a_{n}$ for all $n \geq 1$. By Lemma 1 one has $\delta\left(\gamma_{n}\right)=0$ for all $n \geq 1$. Then the function $F(\lambda):=\delta(\lambda) / d(\lambda)$ is entire in $\lambda$, and it vanishes at infinity. This means that $F(\lambda) \equiv 0$, and consequently, $C(\pi, \lambda) \equiv S^{\prime}(\pi, \lambda)$. Using Lemma 4 from [8] we get $q(x)=q(\pi-x)$ a.e. on $(0, \pi)$.

We will write $a_{n} \in I_{0}$, if the length of the gap $a_{n}$ is equal to zero, and $a_{n} \in I_{1}$, otherwise.

Let us now consider the inverse problem for the periodic BVP $L$. In the general case in Inverse problem 2 we have to specify $\Lambda, \gamma$ and $\Omega$. In the central symmetric case we do not need $\gamma$. On the other hand, the sequence $\Omega=\left\{\omega_{n}\right\}_{n \geq 1}$ does not bring any information because in the central symmetric case $\omega_{n}=0$ for all $n \geq 1$. Unfortunately, in contrast to the separated boundary conditions, for the periodic case the specification of the spectrum $\Lambda$ does not uniquely determine the potential $q$, and we need additional information. For this purpose we introduce the sequence $E=\left\{\xi_{n}\right\}_{n \geq 1}$, where $\xi_{n}=0$ if $a_{n} \in I_{0}, \xi_{n}=1$, if $a_{n} \in I_{1}$ and $\gamma_{n}$ lies at the right endpoint of $a_{n}, \xi_{n}=-1$, if $a_{n} \in I_{1}$ and $\gamma_{n}$ lies at the left endpoint of $a_{n}$. The sequence $E=\left\{\xi_{n}\right\}_{n \geq 1}$ is called the $E$ - sequence for the potential $q(x) \in L_{2}^{\prime}(0, \pi)$. The inverse problem for the periodic BVP $L$ in the central symmetric case is formulated as follows.

Inverse problem 4. Given $\Lambda$ and $E$, construct $q$.

Theorem 7 ([9]). Let $q(x) \in L_{2}^{\prime}(0, \pi)$. Then the specification of $\Lambda$ and E uniquely determines the potential $q$. The solution of Inverse problem 1 can be found by the following algorithm.

Algorithm 1. Given $\Lambda$ and $E$.

(1) Construct $p(\lambda)$ by (5).

(2) Calculate the functions $\Delta(\lambda)=1-p(\lambda)$ and $p^{+}(\lambda)=p(\lambda)-2$. 
(3) Find zeros $\Lambda^{+}=\left\{\lambda_{n}^{+}\right\}_{n \geq 1}$ of $p^{+}(\lambda)$.

(4) Construct $\gamma=\left\{\gamma_{n}\right\}_{n \geq 1}$ as follows: $\gamma_{n}$ lies at the right endpoint of $a_{n}$ if $\xi_{n}=1$; $\gamma_{n}$ lies at the left endpoint of $a_{n}$ if $\xi_{n}=-1$, and $\gamma_{n}=a_{n}$ if $\xi_{n}=0$.

(5) Using $\left\{\gamma_{n}\right\}$ construct the potential $q(x) \in L_{2}^{\prime}(0, \pi)$ by solving Inverse problem 3 .

Denote by $J_{1}$ the set of sequences $E=\left\{\xi_{n}\right\}_{n \geq 1}$ such that $\xi_{n}=0$ if $a_{n} \in I_{0}$, and $\xi_{n}= \pm 1$ if $a_{n} \in I_{1}$. Clearly, if $E$ is the $E$ - sequence for $q$, then $E \in J_{1}$. The next theorem [9] establishes necessary and sufficient conditions for the solvability of Inverse problem 4.

Theorem 8 ([9]). Let real numbers $\Lambda=\left\{\lambda_{n}\right\}_{n \geq 0}$ satisfying (3)-(4) be given. The sequence $\Lambda$ is the spectrum for a certain BVP $L$ with a real potential $q(x) \in L_{2}^{\prime}(0, \pi)$, iff relation (6) holds, where $p(\lambda)$ is constructed via (5). Moreover, if additionally we have a sequence $E=\left\{\xi_{n}\right\}_{n \geq 1} \in J_{1}$, then there exists a unique real function $q(x) \in L_{2}^{\prime}(0, \pi)$ such that $\Lambda$ is the spectrum of $L$, and $E$ is the E-sequence for $q$.

Proof. The necessity is obvious. We will prove the sufficiency. Let real numbers $\Lambda=\left\{\lambda_{n}\right\}_{n \geq 0}$ satisfying (3)-(4) be given. We construct the function $p(\lambda)$ by (5), and calculate the functions $\Delta(\lambda)=1-p(\lambda)$ and $p^{+}(\lambda)=p(\lambda)-2$. Let (6) holds. Then there exist zeros $\Lambda^{+}=\left\{\lambda_{n}^{+}\right\}_{n \geq 1}$ of the function $p^{+}(\lambda)$, and (7) holds. Using (5) by similar arguments as in the proof of Theorem 5 (see also [4]) one gets

$$
p(\lambda)=1-\cos \rho \pi-a \frac{\sin \rho \pi}{\rho}-\frac{\kappa(\rho)}{\rho},
$$

where $\kappa(\rho) \in L_{2}(-\infty, \infty)$ for real $\rho$. Since $p^{+}(\lambda)=p(\lambda)-2$, it follows from (20) that (8) is valid. Let a sequence $E=\left\{\xi_{n}\right\}_{n \geq 1} \in J_{1}$ be given. We introduce real numbers $\gamma=\left\{\gamma_{n}\right\}_{n \geq 1}$ as follows: $\gamma_{n}$ lies at the right endpoint of $a_{n}$ if $\xi_{n}=1 ; \gamma_{n}$ lies at the left endpoint of $a_{n}$ if $\xi_{n}=-1 ; \gamma_{n}=a_{n}$ if $\xi_{n}=0$. Clearly, (9) is valid. We construct the function $d(\lambda)$ by (10), and the sequence $\left\{\alpha_{n}\right\}_{n \geq 1}$ via

$$
\alpha_{n}=\dot{d}\left(\gamma_{n}\right) \Delta\left(\gamma_{n}\right), \quad n \geq 1
$$

Since $\Delta(\lambda)=1-p(\lambda)$, it follows from (20) that

$$
\Delta(\lambda)=\cos \rho \pi+a \frac{\sin \rho \pi}{\rho}+\frac{\kappa(\rho)}{\rho} .
$$

Together with (9) this yields

$$
\Delta\left(\gamma_{n}\right)=(-1)^{n}\left(1+\frac{\kappa_{n}}{n}\right), \quad\left\{\kappa_{n}\right\} \in l_{2} .
$$

Moreover, (13) is valid. It follows from (13), (21) and (22) that (12) holds. It is easy to check that

$$
\operatorname{sign} \dot{d}\left(\gamma_{n}\right)=(-1)^{n}, \quad \operatorname{sign} \Delta\left(\gamma_{n}\right)=(-1)^{n}
$$


In view of (21) and (23) we conclude that $\alpha_{n}>0, n \geq 1$. By Theorem 1 we infer that there exists a unique real potential $q(x) \in L_{2}(0, \pi)$ such that $\left\{\gamma_{n}, \alpha_{n}\right\}_{n \geq 1}$ are the spectral data for the BVP $L_{0}$ for this potential. We construct solutions $C(x, \lambda), S(x, \lambda)$ for Eq. (1) with this potential. Denote

$$
\tilde{\Delta}(\lambda)=\left(C(\pi, \lambda)+S^{\prime}(\pi, \lambda)\right) / 2, \tilde{p}(\lambda)=1-\tilde{\Delta}(\lambda), \tilde{p}^{+}(\lambda)=\tilde{p}(\lambda)-2 .
$$

Using (10) and (21) we get

$$
\Delta\left(\gamma_{n}\right)=\tilde{\Delta}\left(\gamma_{n}\right), \quad n \geq 1
$$

Then the function $F_{0}(\lambda):=(\Delta(\lambda)-\tilde{\Delta}(\lambda)) / d(\lambda)$ is entire in $\lambda$, and it vanishes at infinity. This yields $F_{0}(\lambda) \equiv 0$, i.e. $\Delta(\lambda) \equiv \tilde{\Delta}(\lambda)$, and consequently, $p(\lambda) \equiv \tilde{p}(\lambda), p^{+}(\lambda) \equiv \tilde{p}^{+}(\lambda)$. In particular, this means that the sequence $\Lambda=\left\{\lambda_{n}\right\}_{n \geq 0}$ coincides with the spectrum of the BVP $L$ for the potential $q$. Since $\gamma_{n}$ lies at one of the endpoints of the gap $a_{n}$ for all $n \geq 1$, it follows from Theorem 6 that $q(x) \in L_{2}^{\prime}(0, \pi)$. Now it is clear that $E$ is the $E$-sequence for $q$.

Similar results are valid for other non-separated boundary conditions. For convenience of readers and for completeness of the presentation, we formulate here briefly the main results from [10] related to the boundary conditions

$$
y^{\prime}(0)-a y(0)+b y(\pi)=y^{\prime}(\pi)+a y(\pi)-b y(0)=0 .
$$

We consider the BVP $B$ for the differential equation

$$
-y^{\prime \prime}+q(x) y=\lambda y, x \in(0, \pi), q(x) \in L_{2}^{\prime}(0, \pi)
$$

with the non-separated boundary conditions (24), where $a$ and $b$ are real numbers, $b \neq 0$. Let $\theta(x, \lambda)$ be the solution of Eq. (25) under the initial conditions $\theta(0, \lambda)=1, \theta^{\prime}(0, \lambda)=a$. Eigenvalues $\mu=\left\{\mu_{n}\right\}_{n \geq 0}$ of the BVP $B$ coincide with zeros of the entire function

$$
r(\lambda)=-\theta^{\prime}(\pi, \lambda)-a \theta(\pi, \lambda)+b^{2} S(\pi, \lambda)+2 b .
$$

The eigenvalues $\mu_{n}$ are real, and

$$
\mu_{n}<\mu_{n+2}, \quad \mu_{n}=n^{2}+\pi^{-1}\left(h+(-1)^{n+1} 4 b\right)+\kappa_{n},\left\{\kappa_{n}\right\} \in l_{2},
$$

where $h=4 a+\int_{0}^{\pi} q(t) d t$. The specification of the spectrum $\mu$ uniquely determines the characteristic function $r(\lambda)$ via

$$
r(\lambda)=\pi\left(\lambda-\mu_{0}\right) \prod_{n=1}^{\infty} \frac{\mu_{n}-\lambda}{n^{2}} .
$$

Moreover,

$$
\max _{\lambda \in Q_{n}}|r(\lambda)| \geq|4 b|,
$$


where $Q_{n}=\left[\mu_{2 n}, \mu_{2 n+1}\right]$ if $b>0$, and $Q_{n}=\left[\mu_{2 n-1}, \mu_{2 n}\right]$ if $b<0$. Let $v=\left\{v_{n}\right\}_{n \geq 0}$ be zeros of the entire function $\theta(\pi, \lambda)$. Denote $\eta_{n}=\operatorname{sign}\left(\left|\theta^{\prime}\left(\pi, v_{n}\right)\right|-|b|\right)$. The sequence $\eta=\left\{\eta_{n}\right\}_{n \geq 0}$ is called the $\eta$ - sequence for $B$. The inverse problem is formulated as follows

Inverse problem 5. Given $\mu$ and $\eta$, construct $q, a$ and $b$.

The next theorem gives us the characterization of the spectrum of the BVP $B$.

Theorem 9. For real numbers $\left\{\mu_{n}\right\}_{n \geq 0}\left(\mu_{n} \leq \mu_{n+1}\right)$ to be the eigenvalues of a certain BVP B with real potential $q(x) \in L_{2}^{\prime}(0, \pi)$, it is necessary and sufficient that (26) and (28) hold, where $r(\lambda)$ is constructed by (27).

Denote by $J^{\prime}$ the set of sequences $\eta=\left\{\eta_{n}\right\}_{n \geq 0}$ such that

(i) $\eta_{n}= \pm 1$ if the corresponding zeros of the functions $r(\lambda)$ and $r(\lambda)-4 b$ are simple, and $\eta_{n}=0$ otherwise;

(ii) there exists $N$ (depending on the sequence) such that $\eta_{n}=1$ for all $n>N$.

Clearly, if $\eta$ is the $\eta$ - sequence for $B$, then $\eta \in J^{\prime}$. The next theorem gives us necessary and sufficient conditions for the solvability of Inverse problem 5.

Theorem 10. Let real numbers $\left\{\mu_{n}\right\}_{n \geq 0}\left(\mu_{n} \leq \mu_{n+1}\right)$ satisfying (26) and (28) be given, where $r(\lambda)$ is constructed by (27). Then for each sequence $\eta \in J^{\prime}$ there exists a unique real function $q(x) \in L_{2}^{\prime}(0, \pi)$ and real numbers $a$ and $b$ such that $\mu=\left\{\mu_{n}\right\}_{n \geq 0}$ is the spectrum of $B$, and $\eta$ is the $\eta$ - sequence for $B$.

We note that in [17] stability of the solution of the inverse problem for the BVP $B$ is established. Some other results related to the inverse problem theory for the Sturm-liouville operators can be found in [18]-[20] and other works.

\section{Acknowledgement}

This work was supported by Grant 17-11-01193 of the Russian Science Foundation.

\section{References}

[1] V. A. Marchenko, Sturm-Liouville operators and their applications, Naukova Dumka, Kiev, 1977; English transl., Birkhäuser, 1986.

[2] B. M. Levitan, Inverse Sturm-Liouville problems. Nauka, Moscow, 1984; English transl., VNU Sci. Press, Utrecht, 1987.

[3] J. Pöschel and E. Trubowitz, Inverse Spectral Theory, Academic Press, New York, 1987.

[4] G. Freiling and V. A. Yurko, Inverse Sturm-Liouville Problems and their Applications. NOVA Science Publishers, New York, 2001. 
[5] V. A. Yurko, Method of Spectral Mappings in the Inverse Problem Theory. Inverse and Ill-posed Problems Series. VSP, Utrecht, 2002.

[6] I. V. Stankevich, An inverse problem of spectral analysis for Hill's equation, Doklady Akad. Nauk SSSR, 192 (1970), 34-37. (in Russian); English transl. in Soviet Math. Dokl., 11 (1970), 582-586.

[7] V. A. Marchenko and I. V. Ostrovskii, A characterization of the spectrum of the Hill operator, Mathem. Sb., 97 (1975), 540-606 (in Russian); English transl. in Math. USSR-Sb., 26 (1975), 493-554.

[8] V. A. Yurko, An inverse problem for second order differential operators with regular boundary conditions, Matem. Zametki, 18 (1975), 569-576 (in Russian); English transl. in Mathematical Notes, 18 (1975), 928-932.

[9] V. A. Yurko, On a periodic boundary value problem, Differ. Equations and Theory of Functions, Saratov Uni., Saratov, 1981, 109-115 (in Russian).

[10] V. A. Yurko, On recovering differential operators with nonseparated boundary conditions, Study in Math. and Appl., Bashkir Uni., Ufa, 1981, 55-58 (in Russian).

[11] O. A. Plaksina, Inverse problems of spectral analysis for the Sturm-Liouville operators with nonseparated boundary conditions, Mathem. Sb., 131 (1986), 3-26 (in Russian); English transl. in Math. USSR-Sb. 59 (1988), $1-23$.

[12] I. M. Guseinov, M. G. Gasymov and I. M. Nabiev, An inverse problem for the Sturm-Liouville operator with nonseparable self-adjoint boundary conditions, Sib. Mathem. Zh., 31 (1990), 46-54 (in Russian); English trans. in Siberian Math. J., 31 (1990), 910-918.

[13] I. M. Guseinov and I. M. Nabiev, Solution of a class of inverse boundary-value Sturm-Liouville problems, Mathem. Sb., 186 (1995), 35-48 (in Russian); English transl. in Sbornik: Mathematics, 186 (1995), 661-674.

[14] P. Kargaev and E. Korotyaev, The inverse problem for the Hill operator, a direct approach, Invent. Math., 129 (1997), 567-593.

[15] V. A. Yurko, On differential operators with nonseparated boundary conditions, Funkt. Analiz i Prilozh., 28 (1994), 90-92 (Russian); English transl. in Functional Analysis and Applications, 28 (1994), 295-297.

[16] V. A. Yurko, The inverse spectral problem for differential operators with nonseparated boundary conditions, Journal of Mathematical Analysis and Applications, 250 (2000), 266-289.

[17] G. Freiling and V. A. Yurko, On the stability of constructing a potential in the central symmetry case, Applicable Analysis, 90 (2011), 1819-1828.

[18] G. Freiling and V. A. Yurko, Inverse problems for Sturm-Liouville equations with boundary conditions polynomially dependent on the spectral parameter, Inverse Problems, 26 (2010), 055003, 17pp.

[19] Y. P. Wang, Inverse problems for discontinuos Sturm-Liouville operators with mixed spectral data, Inverse Problems in Sci. Eng., 23 (2015), 1180-1198.

[20] C. T. Shieh and V. A. Yurko, Inverse spectral and nodal problems for discontinuous boundary value problems, Journal of Mathematical Analysis and Applications, 347 (2008), 266-272.

Department of Mathematics, Saratov University, Astrakhanskaya 83, Saratov 410012, Russia.

E-mail: yurkova@info.sgu.ru 